\title{
Planar array pattern null steering by controlling the side elements
}

\author{
Zhong-Hui Zhao ${ }^{\dagger}$ and Hui-Ling Zhao and Yong-Chao Xu \\ Department of Electronics and Information, \\ Northwestern Polytechnical University, Xi'an, China \\ ${ }^{\dagger}$ E-mail: zhaozhh@mail.nwpu.edu.cn \\ www.nwpu.edu.cn
}

\begin{abstract}
In this article, a new planar array nulling approach which only adjusts the phase of the side elements was proposed. In this approach, pattern nulling problem was modeled as a constraint problem and solved by $\varepsilon$-self-adaptive differential evolution algorithm. Simulation results show that the proposed method successfully design patterns with preferred nulling level, sidelobe level and stable desired steering direction.

Keywords: Planar Array Nulling; $\varepsilon$-Self-Adaptive Differential Evolution; Side Elements; Phase Only.
\end{abstract}

\section{Introduction}

The main aim of null steering algorithm is to steer the mainlobe of an antenna array to the desired direction and place a notch in the interfering signal direction [1]. Measurements to achieve null steering include adjusting the weights (excitation amplitude and phase), phase only, amplitude only and controlling the element position.

For phased array, phase only method is more economic than others in practical applications[2]. Algorithms such as GA[3] and CVX[4] have already been used in phase only beamforming. In literature [5], the author applied SADE to the synthesis of rectangular array and illustrated that SADE was better than DE and PSO in dealing with this problem.

When calibrating each element in an array, the array will achieve ideal beamforming results. But it is time consuming especially for large arrays. In order to decrease the complexity, literature [6] suggested that only part of the elements in the array were used to array adaptive nulling. In [7], the phase and amplitude excitations of the boundary elements were adjusted to get lower sidelobes.

In this paper, the planar array nulling problem is modeled by a constraint optimization problem in which only the phase excitations of part of the 
boundary elements is adjusted while the rest of elements keeping unchanged. Then $\varepsilon$-self-adaptive differential evolution is applied to solve the problem. Experiments show that, the proposed method can successfully design patterns with desired nulls, near sidelobe level and steering directions by adjusting only part of the boundary elements' phases.

\section{Problem Definition}

Given the weighting vector $w=\left[w_{1}, w_{2}, \cdots, w_{N}\right]$ of an N-element planar array, the array's amplitude pattern $A F(\theta, \varphi)$ is given by

$$
A F(\theta, \varphi)=\left|w^{H} a(\theta, \varphi)\right|
$$

where $a(\theta, \varphi)=\left[a_{1}(\theta, \varphi), a_{2}(\theta, \varphi), \ldots, a_{N}(\theta, \varphi)\right]$ is the steering vector with $a_{n}(\theta, \varphi)=e^{-j \psi_{n}(\theta, \varphi)}, j=\sqrt{-1} . \psi_{n}(\theta, \varphi)=\frac{2 \pi}{\lambda}\left(x_{n} \sin \theta \cos \phi+y_{n} \sin \theta \sin \phi\right)$ is the propagation phase difference depends on the array plane and $\lambda$ is the wavelength in free space.

If we adjust the weights of boundary elements, the beam pattern will also be changed which may lead to higher sidelobe levels and changes on the steering direction. In order to eliminate these effects, the pattern nulling problem can be described by the following constraint optimization problem,

$$
\begin{array}{lll}
\min \max & |S L L| \\
\text { subject to } & \max \left|A F_{\text {null }}\right|-\delta_{n} \leq 0 & 1 \\
& \max \left|A F_{\text {nearsLL }}\right|-\delta_{s} \leq 0 & 2 \\
& \left|\mathrm{~A} F\left(\theta_{m}\right)\right|-\max |A F| \leq 0 & 3
\end{array}
$$

where SLL is the sidelobe level. The fitness function is to minimize the maximum sidelobe level under Constraint 1,2 and 3. Constraint 1 and 2 describes the maximum nulls level of array pattern and the maximum near sidelobe level at specified directions no larger than $\delta_{\mathrm{n}}$ and $\delta_{s}$, respectively. The last constraint is to force keeping the mainlobe direction, which is of great importance in beamforming or direction of arrival.

\section{3.\&-Self-adaptive Differential Evolution Overview}

The DE algorithm is sensitive to the setting of control parameters when considering effectiveness, efficiency and robustness of the algorithm [8]. Better control parameters lead to better individuals and more likely to generate better 
offsprings. DE algorithm will convergence faster for adaptive parameters setting than fixed parameters setting [5].

Crossover control parameter (CR) and mutation scale factor (F) are calculated with the following equations,

$$
\begin{gathered}
C R_{i, G+1}= \begin{cases}\operatorname{rand}(0,1) & \text { if } \operatorname{rand}(0,1) \leq \tau_{2} \\
C R_{i, G} & \text { otherwise }\end{cases} \\
F_{i, G+1}= \begin{cases}F_{l}+\operatorname{rand}(0,1) \cdot F_{u} & \text { if } \operatorname{rand}(0,1) \leq \tau_{1} \\
F_{i, G} & \text { otherwise }\end{cases}
\end{gathered}
$$

Where $F_{l}, F_{u}, \tau_{1}$ and $\tau_{2}$ are the fixed values and $\tau_{1}, \tau_{2}$ represents possibilities of controlling of $\mathrm{F}$ and $\mathrm{CR}$.

It has been noticed that the performance of DE mainly depends on the mutation strategy. Therefore in order to improve the performance, here combining different mutation strategies,

$$
\text { strategy }= \begin{cases}{ }^{\prime} D E / \text { best } / 2^{\prime} & \text { if }(G+i) \bmod 5=3 \& G>0.8 G_{\max } \\ x_{r_{5}, G}+F \bullet\left(x_{\text {best }, G}-x_{i, G}\right)+F \bullet\left(x_{r_{1}, G}-x_{r_{2}, G}\right) & \text { if }(G+i) \bmod 5=1 \& G>0.4 G_{\max } \\ ' D E / \text { rand } / 1^{\prime} & \text { otherwise }\end{cases}
$$

where 'mod' denotes the modulo operation, $G$ and $G_{\max }$ are the iteration process number and maximum number of iteration respectively. Vector $x_{b e s t, G}$ and $x_{i, G}$, is the best vector and current vector in generation $G . r_{d}, d=1,2,3$ present the number generated within the range $[1, N P]$ and also different from the index $i$.

The selection operation is different from other unconstraint DE algorithm as it has to deal with the constraints. The constraint violence is a useful way to deal with constraint. Here define all constraint violences $(v)$ as the sum of all constraints and in the algorithm it will be set to zero when the constraints satisfy the equation. The selecting operation of the algorithm distinguishes between feasible $(v=0)$ and infeasible individuals: any feasible solution is better than any infeasible one.

\section{SRG-based Mainlobe Region Determation}

To determine the sidelobe region, SRG (Segment Region Growing) method is used to discriminate the boundary between the mainlobe region and the sidelobe region [9]. The basic idea of SRG method is starting at a seed point, and then adds adjacent pixels with similar property from the growth zones, which will be a new starting point until no more new pixel is added. 
The peak point of the pattern is chosen as the initial point. Every seed point has to compare with the neighbor point to check its property by a stop criterion

$$
A F_{m, l}-A F_{m+p, l+q} \geq 0
$$

where $(p, q) \in\{(-1,0),(1,0),(0,-1),(0,1)\}$ represents the four growing directions. The procedure will not stop until the mainlobe region cannot grow again.

\section{Experiment Setup and Results}

Consider a $9 * 9$ square planar array with one-half wavelength spaced in both $\mathrm{x}$ and $y$ directions as shown in Fig.2, where 'o'denotes the main array with Chebyshev weighted and only the ' $\bullet$ ' elements are adjusted to form nulls in the simulation.

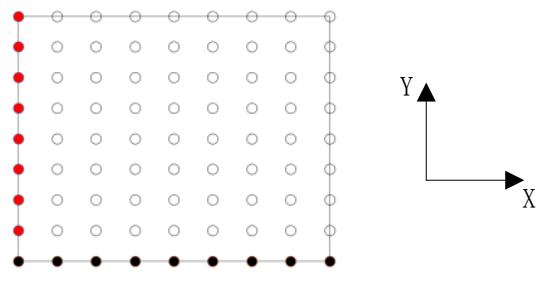

Fig.1. Geometry of the proposed planar array

As design constraints, we set near sidelobe (zones surrounding the main lobe within 15 degree) level below $-18 \mathrm{~dB}$ and null level no more than $-35 \mathrm{~dB}$. The population size, $G_{\max }$, initial F, initial CR, $F_{l}, F_{u}, \tau_{1}$ and $\tau_{2}$ are chosen as 136, 200,0.3,0.3,0.1,0.9,0.1 and 0.1, respectively.

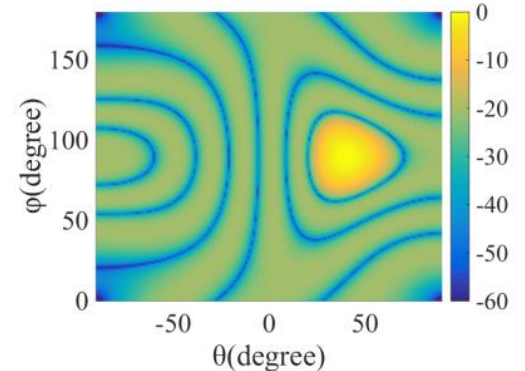

Fig. 2a. -20dB Chebyshev antenna pattern

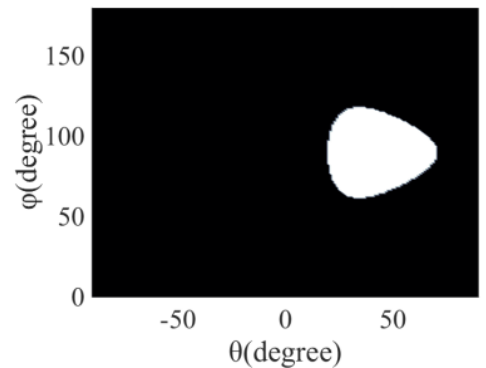

Fig.2b. The SRG results

The main array pattern with $-20 \mathrm{~dB}$ weighted Chebyshev steering at $\left(130^{\circ}, 90^{\circ}\right)$ is in shown in Fig.2a, and the vacuum in Fig.2b illustrates the mainbeam area of the array pattern segregated by SRG. 


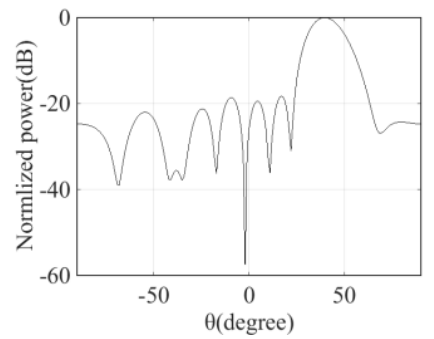

Fig. 3a. Antenna pattern when $\varphi=90^{\circ}$ with a at $\theta\left(-42^{\circ} \sim-35^{\circ}\right)$

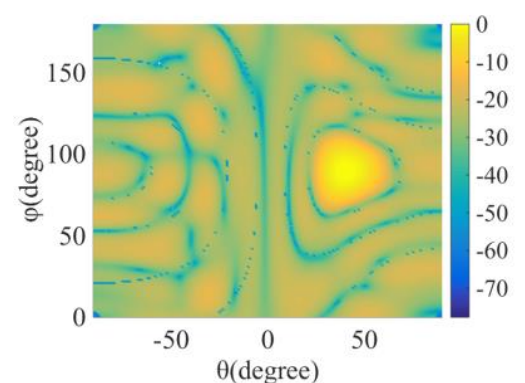

Fig.4. Pattern nulling with a wide null in two planes imposed at $\theta\left(-40^{\circ} \sim-37^{\circ}\right)$ and $\varphi\left(50^{\circ} \sim 52^{\circ}\right)$ respectively

The Fig.3 shows the algorithm's ability and converge performance of creating wide null in the interference direction while keeping the main lobe direction and near sidelobe level under the constraints.

The Fig.4 shows the described planar array can also form nulls in two planes.

\section{Conclusion}

A constraint optimization method for the planar array null steering using side elements and phase only control has been presented. The excited phases were computed using $\varepsilon$-self-adaptive differential evolution. The simulation results show that the proposed method can effectively form nulls with constraint characteristics. Also the work can be applied to other arrays, such as linear array and circular array with other initial weights (i.e., Uniform, Taylor ).

\section{Acknowledgement}

This work is supported by 20142053025 of aeronautical science foundation of China. 


\section{References}

1. Ghose, R. N. Electronically adaptive antenna systems. Antennas \& Propagation IEEE Transactions on, 12(2), 161-169(1964).

2. Baird, C. A., \& Rassweiler, G. G. Adaptive sidelobe nulling using digitally controlled phase-shifters. IEEE Transactions on Antennas \& Propagation, 24(5), 638-649(1976).

3. Haupt, R. L. Phase-only adaptive nulling with a genetic algorithm. IEEE Transactions on Antennas \& Propagation, 45(6), 1009-1015(1997).

4. Kajenski, P. J. Phase only antenna pattern notching via a semidefinite programming relaxation. IEEE Transactions on Antennas \& Propagation, 60(5), 2562-2565(2012).

5. Chatterjee, A., \& Mahanti, G. K. Combination of fast fourier transform and self-adaptive differential evolution algorithm for synthesis of phase-only reconfigurable rectangular array antenna. annals of telecommunications annales des télécommunications,69(9-10), 515-527(2013).

6. Chapman, D. Partial adaptivity for the large array. IEEE Transactions on Antennas \& Propagation, 24(5), 685-696(1976).

7. Mohammed, J. R., \& Sayidmarie, K. H. Sidelobe cancellation for uniformly excited planar array antennas by controlling the side elements. IEEE Antennas \& Wireless Propagation Letters, 13(13), 987-990. (2014).

8. Mezura-Montes, E. Constraint-handling in evolutionary optimization. (38(11), 1989-95, 2009).

9. Liu, Y., Chen, S. L., Zhang, L., \& Liu, Q. H. Determining the first-null mainlobe region of an arbitrary pattern for $2 \mathrm{~d}$ numerical pattern synthesis algorithm. IEEE Transactions on Antennas \& Propagation, 64(3), 1-1. (2016). 